\title{
ANALYSIS OF PRESSURE BEHAVIOR IN A TEMPERATURE CONTROLLED MOLECULAR DYNAMIC FLOW
}

\author{
Hamed R. Najafi, S.M. Hossein Karimian \\ Department of Aerospace Engineering, Amirkabir University of Technology, Tehran, Iran \\ e-mail: hamedramezani@aut.ac.ir; hkarim@aut.ac.ir
}

\begin{abstract}
Thermo-fluid properties are required for numerical modeling of nano/micro devices. These properties are mostly obtained from results of molecular dynamics (MD) simulations. Therefore, efforts have been put in developing methods for numerical evaluation of fluid properties, such as pressure. In this paper, the pressure behavior in a controllable nanochannel flow is investigated. The nanoflow field is created by imposing a gradient of a macroscopic property such as temperature. Details of the pressure calculation method in a molecular system and its sensitivity to the approximations made are described first. The effect of temperature rise in a uniform flow on the pressure field is studied next. Then, in the flow under a fixed mean velocity condition, the effect of temperature gradient as a controllable property on the pressure field of nanoflow is studied. Velocity, pressure and molecular density of nanoflows with various temperature gradients and different temperature levels are investigated as well. It has been found that the temperature level at which the temperature gradient is imposed, is important. A fixed temperature gradient will not always lead to the same pressure gradient at different temperature levels. Furthermore, quite interestingly, it is observed that at a fixed temperature gradient, with the variation of mean velocity the pressure field also varies.
\end{abstract}

Keywords: nanofluid, molecular dynamics, pressure, bin size, sampling, periodic flow

\section{Introduction}

Flow behavior at micro and nano scales has been a subject of interest in the recent years. The flow in a nanochannel, as a typical reduced-size fluid flow system, can demonstrate different aspects of nanofluid systems. This is why many studies can be found in literature focusing on the simulation of nanochannel flows (Mi and Chwang, 2003).

Molecular Dynamics (MD) is a deterministic method to calculate position of molecules and their dynamic properties. Different potential functions have been introduced to represent molecular forces in MD simulations (see for example Leach, 1999; Sadus, 2002). Extracting macroscopic properties such as velocity, temperature and pressure from microscopic data has been also a challenging issue for scientists (Allen and Tildesley, 1987; Karniadakis et al., 2002; Karimian et al., 2011). In earlier studies, the boundary of the domain was not of primary importance and simple. Periodic boundary conditions were used (Stillinger and Rahman, 1974; Travis and Evans, 1977; Koplik et al., 1989; Travis and Gubbins, 2000; Nagayama and Cheng, 2004). Implementation of boundary conditions in MD simulation has been the center of attention in the recent years, especially in the field of mechanics (Sun and Ebner, 1992; Huang et al., 2004, 2006; Hanasaki and Nakatani, 2006). In addition to the inlet/outlet boundary conditions, different approaches exist in the literature to implement wall boundary conditions in molecular dynamics. The most usual choice is to model walls by two rows of molecules. Wall molecules are allowed to oscillate about their initial positions at which they are fixed. This is done either by assigning heavy weights to the wall molecules (Koplik et al., 1988), rescaling the velocity of wall molecules to their initial values (Huang et al., 2004) or using fictitious springs (Fan et al., 2002; Huang et 
al., 2006; Sofos et al., 2009; Branam and Micci, 2009; Kamali and Kharazmi, 2011). In addition to these, some studies can be found where the walls were modeled differently. For example, see the work of Ziarani and Mohamad (2005), where the walls were modeled as reflective. Since wall boundary conditions are not the subject of this paper, interested scientists are encouraged to read the above-mentioned references.

Boundary conditions at the inlet and outlet have been also a subject of research. With proper boundary conditions, the desired flow field can be created within a nanochannel. There are different methods in the literature to create controllable nanoflow. These methods are classified in four categories. Creating a flow with an external force or acceleration to move molecules in a specified direction is the most simple and straightforward method. Note that the extra energy added to the flow field due to external force must be taken out of the domain to hold the energy balance (Koplik et al., 1988; Fan et al., 2002; Ziarani and Mohamad, 2005; Sofos et al., 2009; Kamali and Kharazmi, 2011).

Another method found in the literature makes use of motion of a piston or plate upstream of the inlet to create the flow. In this method, the solution domain is extended to provide extra space between the piston and the inlet. To control flow condition in the outlet as well, sometimes a piston and the required extra space is added to the end of the solution domain. In this case, there is no need to implement periodic boundary conditions or insert and delete molecules at the inlet or outlet of the solution domain. This approach works well for dense systems. Since additional molecules are to be taken into account in these added spaces, extra computational effort is required in these methods. The other disadvantage is that the solution is limited to the time in which the piston moves in the space between its initial position and the inlet/outlet (Huang et al., 2004, 2006; Hanasaki and Nakatani, 2006).

The third method involves using a temperature gradient to create a nanoflow. In this approach, hot and/or cold walls are embedded within the domain along the flow direction (Han, 2008; Liu and Li, 2010; Darbandi et al., 2011). The role of the temperature gradient is to change the kinetic energy of the fluid. At high temperatures, the kinetic energy and therefore the pressure of the fluid increase. This pressure gradient causes the fluid to move downstream. The energy balance can be established between hot and cold plates to prevent accumulation of extra energy in the domain. It should be noted that in practice, hot or cold temperature walls cannot be easily mounted within the flow and, therefore, implementation of this approach is not straightforward.

The fourth method creates a flow by wall motion. In this approach, walls of a channel move in opposite or parallel directions with a constant velocity to create the flow (Zhang et al., 2009; Kim et al., 2010).

While all of the above-mentioned methods achieve the goal of creating a molecular flow in nanochannels, they fail to offer a proper approach to produce the predefined flow. The goal of the present research is to investigate pressure behavior in a controllable nanochannel flow created by a gradient of a macroscopic property such as temperature. In the following sections, we first introduce the calculation method used here to evaluate pressure in a nanoflow. Then we will discuss details about the nanoflows controlled by the temperature gradient, especially the behavior of pressure.

\section{Molecular dynamics}

In molecular dynamics, positions of molecules are determined using Newton's second law. Intermolecular fluid-fluid forces are calculated using the Lennard-Jones 12-6 potential equation (Rapaport, 2004)

$$
U_{i j}=4 \varepsilon\left[\left(\frac{\sigma}{r_{i j}}\right)^{12}-\left(\frac{\sigma}{r_{i j}}\right)^{6}\right]
$$


In this equation, $U_{i j}$ is the Lennard-Jones potential, $r_{i j}$ is the distance between two interacting molecules $i$ and $j, \varepsilon$ is the energy scale, and $\sigma$ is the finite distance at which the intermolecular potential is zero. The derivative of the L-J potential with respect to $r_{i j}$ represents the intermolecular force

$$
f_{i j}=-\frac{d U_{i j}}{d r_{i j}} \quad f_{i j}=\frac{48 \varepsilon}{r_{i j}^{2}}\left[\left(\frac{\sigma}{r_{i j}}\right)^{12}-\frac{1}{2}\left(\frac{\sigma}{r_{i j}}\right)^{6}\right] r_{i j}
$$

In the above equation, $f_{i j}$ is the force of molecule $j$ acting on molecule $i$. It is common to enforce this equation within a cut-off distance to reduce the computational cost. We chose a cut-off distance of $r_{c}=2.5 \sigma$ herein, which is usually used in other references to simulate the argon flow (Koplik et al., 1989; Priezjev, 2007; Sofos et al., 2009).

\section{Pressure formula}

In a macroscopic analysis, pressure is calculated solely based on the virial equation of state. In a microscopic system however, pressure is calculated from the virial equation of state on all atoms plus summation of intermolecular forces multiplied by corresponding distances between them. For pair-additive force fields, the fluid pressure $P$ can be estimated through the virial equation of state given below (Allen and Tildesly, 1987)

$$
P=\frac{N k_{B} T}{V}+\frac{1}{3 V} \sum_{i=1}^{N} \sum_{j>i}^{N} r_{i j} f_{i j}
$$

where $N$ is the number of molecules in the computational domain, $k_{B}$ is Boltzmann's constant, and $V$ and $T$ are volume and temperature of the computational domain, respectively. The first term in Eq. (3.1) is called the kinetic term and contains temperature of the computational domain. In a microscopic view, temperature is defined as

$$
T=\frac{m}{3 N k_{B}} \sum_{n=1}^{N} \sum_{i=1}^{3}\left(V_{n_{i}}-\bar{V}_{i}\right)^{2}
$$

where $n$ denotes the molecule number in the domain, $i=1,2,3$ denote the $x, y$, and $z$ components of the atomic velocity $\mathbf{V}_{n}, \bar{V}_{i}$ is the $i$-th component of the mean flow velocity $\mathbf{V}$, and $m$ is the atom mass. The mean velocity can be obtained from any of the several averaging methods like SAM (Tysanner and Garcia, 2004), CAM (Tysanner and Garcia, 2005) or SMC (Karimian et al., 2011) that reduces statistical errors in its calculation. In this paper, the SMC method is used to calculate the mean velocity. As can be observed in Eq. (3.2), temperature is related to the kinetic energy of molecules in the microscopic view, which is always positive. The velocity difference within parentheses that represents instantaneous velocity due to thermal fluctuations is called the virial velocity.

The second term in Eq. (3.1) includes the effect of forces between the molecules; this term is called the potential term. The kinetic term is always positive but the potential term may be positive or negative. A zero force exists between two molecules at a distance of $2^{1 / 6}$ based on the L-J potential model. If the distance between two molecules is higher than this value, the sign of the pair-additive force will be negative and the molecules attract each other. On the other hand, when the distance between two molecules is less than this value, the force is of repellant nature.

The pressure calculation method defined by Eqs. (3.1) and (3.2) is implemented in an inhouse, MD code to determine pressure values from the results of MD simulations. Please note that this code is used in authors' previous works in which its accuracy in calculation of macroscopic properties were demonstrated (Karimian et al., 2011; Karimian and Namvar, 2012; Namvar and Karimian, 2012; Karimian and Izadi, 2013). 


\section{Pressure calculation}

The purpose of this Section is to validate the pressure calculation. According to Eq. (3.1), pressure has two parts, kinetic and potential terms. Our experience shows that precautions should be taken in calculation of pressure in MD simulation. This is demonstrated by considering a cubic region with its molecules in equilibrium, which is a constant Number, Volume and Energy (NVE) simulation. We know that pressure would be the same all over this region. The size of this cube is $L=45 \AA$ and it contains $N=1728$ mono atomic noble gas molecules of Argon. Atomic diameter of Argon is $\sigma=3.4 \AA$ and its energy parameter is $\varepsilon=1.67 \cdot 10^{-21} \mathrm{~J}$. A periodic boundary condition is applied in all directions. Argon molecules are initially arranged in a lattice form of Face-Centered Cube (FCC). MD simulation of Argon molecules starts from the initial temperature of $120 \mathrm{~K}$, and the mean velocity of zero. The equation of motion is integrated over time with $\Delta t=10^{-15} \mathrm{~s}$, using Verlet's scheme (Verlet, 1967). The number of molecules is constant during the simulation. Macroscopic properties at any point of the molecular domain is extracted via sampling and averaging of molecular properties within the control region around that point, called a bin (Kamiadakis et al., 2002). Four bins with different sizes are selected for sampling and averaging of flow properties. As shown in Fig. 1, the largest bin covers the whole cube and contains all argon molecules. Other bins are smaller cubes with lateral sizes of $3 / 4,1 / 2$ and $1 / 4$ of the lateral size of the whole domain. In volumetric fraction, these cubes are $27 / 64,1 / 8$, and $1 / 64$ of the whole domain, respectively. Note that the periodic boundary condition is applied on the cube boundaries. Therefore, similar to other three bins, bin $1 / 1$ is also simulated as if it is in the middle of the domain. Obviously, pressure obtained from all bin sizes should be equal. The molecular dynamics simulation is continued for 30000 time steps of $10^{-15} \mathrm{~s}$, when equilibrium is reached. Although pressure as a macroscopic property should be independent of the bin size, the results show that different pressure values are obtained. The origin of this difference is in the potential part of the pressure equation.

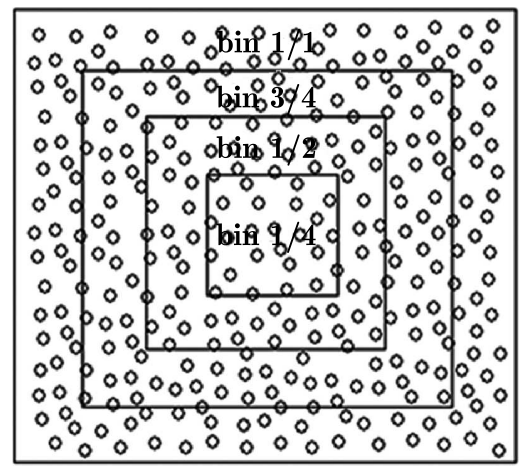

Fig. 1. Bin sizes for pressure calculation in a periodic stationary molecular flow

The kinetic part of pressure, which is solely based on the properties of atoms and is independent of molecular interactions, converges to the value of $27 \mathrm{MPa}$ in all bins with different sizes. The potential part of pressure, however, converges to different values of $-22.3,-18.4,-1.2$, and $5.9 \mathrm{MPa}$ in bins with sizes of $1 / 1,3 / 4,1 / 2$, and $1 / 4$, respectively. This part of pressure is calculated from summation of all pair-additive forces between molecules multiplied by the distance between them. In this approach, no cut-off distance is considered for the molecules. More numerical tests have shown that inclusion of the cut-off distance cannot resolve this problem of pressure calculation, unless the influence of the molecules outside the calculation bin within the cut-off distance from its boundaries is taken into account. Having implemented this, calculated pressures in all of the bins converge to the value of $31.4 \mathrm{MPa}$ at the equilibrium state with minor pressure oscillations and differences in bins with sizes of $1 / 4$ and $1 / 2$ due to the bin size. Note 
that the number of molecules within a bin plays an important role in calculation of pressure. More details about the effect of bin size on sampling and averaging can be found in (Karimian and Izadi, 2013). Calculation of pressure in bin 1/1 with LAMMPS (Plimpton, 1995) converged to the same value with less than a 3 percent difference, which verifies the present method for calculation of pressure in MD simulation.

\section{Control of pressure by temperature}

As noted, macroscopic properties can be extracted from microscopic properties of molecules, calculated by MD simulation. In contrast to this, here we would like to set a macroscopic property in the solution domain. For this purpose, consider a periodic flow in a nanochanel with length of $L=144.6 \AA$ and cross-section of $36.15 \AA \times 36.15 \AA$. This flow contains $N=940$ mono-atomic noble gas molecules of Argon. Periodic boundary conditions are applied on the boundaries of the domain. Argon molecules are initially arranged in a FCC lattice form, and solution starts from the initial temperature and velocity of $120 \mathrm{~K}$ and $5 \mathrm{~m} / \mathrm{s}$, respectively. As shown in Fig. 2, the solution domain is divided into 8 equal bins. We are going to set a desired pressure within the domain and study its effects on the solution; but before that, the solution is proceeded for 400000 time steps of $\Delta t=10^{-15} \mathrm{~s}$ to reach equilibrium. During the solution, temperature of $120 \mathrm{~K}$ and mean velocity of $5 \mathrm{~m} / \mathrm{s}$ are continuously enforced in the first bin. At each time step, the mean velocity in this bin is updated by adding the difference between desired and calculated mean velocities to the velocity of each molecule inside the bin. In a similar fashion, at each time step, temperature in the bin is updated by rescaling the virial velocity of molecules. As expected, temperature and flow velocity within the whole solution domain reach $120 \mathrm{~K}$ and $5 \mathrm{~m} / \mathrm{s}$ respectively, at the equilibrium. The averaging method of SMC (Karimian et al., 2011) is applied to calculate pressure using Eq. (3.1) at each time step. Solution convergence for velocity and pressure in all 8 bins is shown in Fig. 3.

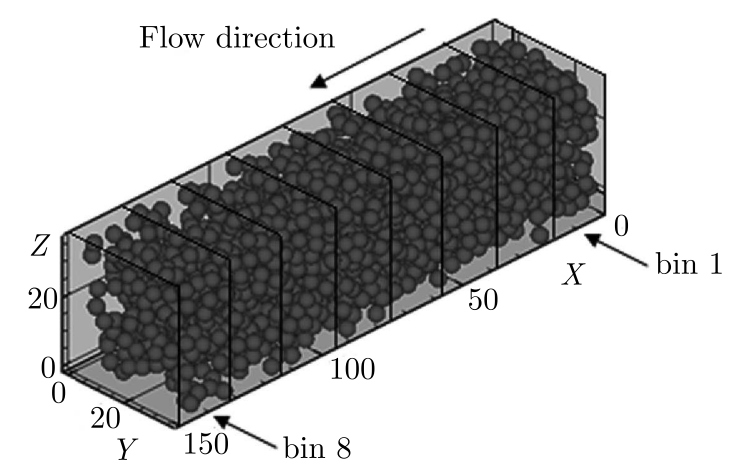

Fig. 2. Periodic flow including 940 molecules with 8 bins along the $x$ direction

As expected, the mean velocity has converged to a value of $5 \mathrm{~m} / \mathrm{s}$ in all of the bins. The convergence of the mean velocity values is faster than that for the pressure. We believe that this is because the mean velocity is weakly dependent on interactions between molecules. In Fig. 3b, pressure has converged to a value of $2.9 \mathrm{MPa}$.

Now we would like to set a new value for pressure in the solution domain. For a fixed number of molecules, pressure can be set to a new value by changing temperature of molecules. As noted above, temperature can be set by rescaling the virial velocity of molecules. To examine this, temperature is raised up from $120 \mathrm{~K}$ to $150 \mathrm{~K}$ in the first bin of this periodic flow at time $4 \cdot 10^{-10} \mathrm{~s}$; i.e. in the time step of 400001 . Then solution is continued for an extra 400000 time steps. The enforced mean velocity is still $5 \mathrm{~m} / \mathrm{s}$ in the first bin. Analysis of the solution shows that pressure increases in the first bin and consequently propagates to the rest of the periodic 
(a)

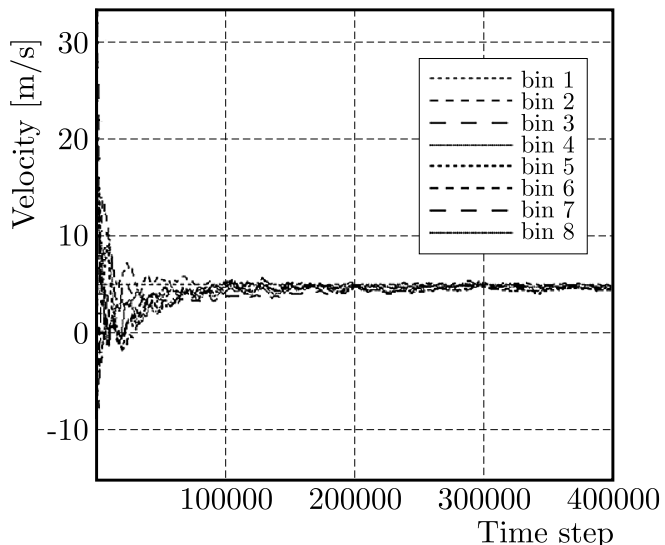

(b)

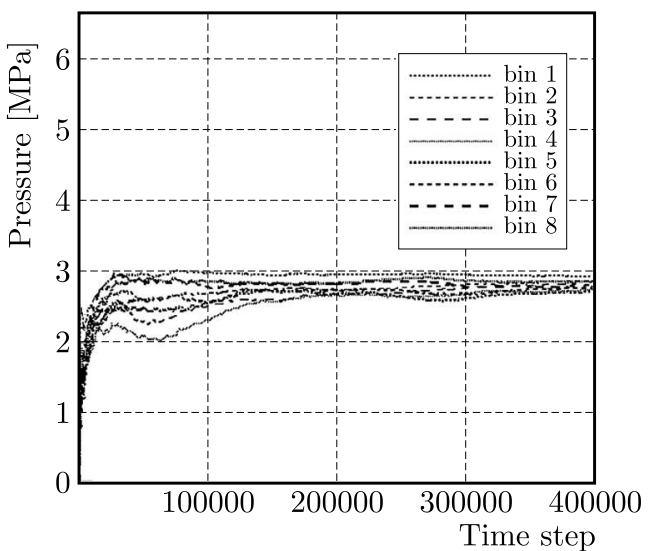

Fig. 3. Solution convergence in bins for: (a) velocity in a periodic flow with enforced velocity of $5 \mathrm{~m} / \mathrm{s}$ in bin 1, and (b) pressure in a periodic flow with enforced temperature of $120 \mathrm{~K}$ in bin 1

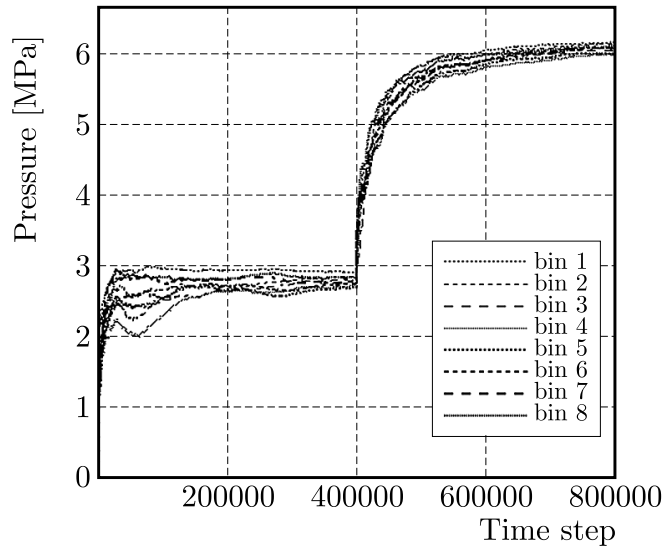

Fig. 4. Convergence of pressure in different bins of the periodic flow with the mean flow velocity of $5 \mathrm{~m} / \mathrm{s}$. A raise in temperature from $120 \mathrm{~K}$ to $150 \mathrm{~K}$ is enforced in the first bin at the time step 400001

flow until the equilibrium is reached. The convergence of pressure in all of the bins is shown in Fig. 4. At the equilibrium, pressure reaches $6.1 \mathrm{MPa}$ all over the solution domain. Again, the mean flow velocity also reaches the value of $5 \mathrm{~m} / \mathrm{s}$.

In the next step, we would like to see how effectively the temperature gradient along a periodic flow can alter pressure, or how quick would be the response of pressure to the temperature gradient along the flow. In the first 400000 time steps, the flow field reaches its equilibrium for the enforced temperature and mean flow velocity of $120 \mathrm{~K}$ and $5 \mathrm{~m} / \mathrm{s}$, respectively, in the first bin. Then, at the time step 400001, a temperature difference of $30 \mathrm{~K}$ is applied between bins 3 and 7 , by setting temperature values to $180 \mathrm{~K}$ and $150 \mathrm{~K}$, respectively.

During the first 400000 time steps, SMC averaging uses all solution data from the first time step. However, after the implementation of temperature change at the time step 400001, we cannot include much data from the previous time steps in SMC averaging. If we start SMC averaging promptly from the time step 400001, undesirable pressure fluctuations will appear in the results. In this case, noticeable extra time steps would be required to resolve this inconsistency in SMC averaging. Our experience shows that very good results can be obtained if only a small portion of data before the time step at which the temperature change is applied (i.e. 400001), is involved in SMC averaging. Here, pressure data of 30 time steps are enough. This means that SMC averaging uses the data from the time step 399971 and after that. Convergence rates of pressure in all of the bins are shown in Fig. 5. As can be seen, pressure increases from $2.9 \mathrm{MPa}$ at temperature of $120 \mathrm{~K}$ to $7.90 \mathrm{MPa}$ and $7.15 \mathrm{MPa}$ at temperatures of $180 \mathrm{~K}$ and $150 \mathrm{~K}$ in bins 3 

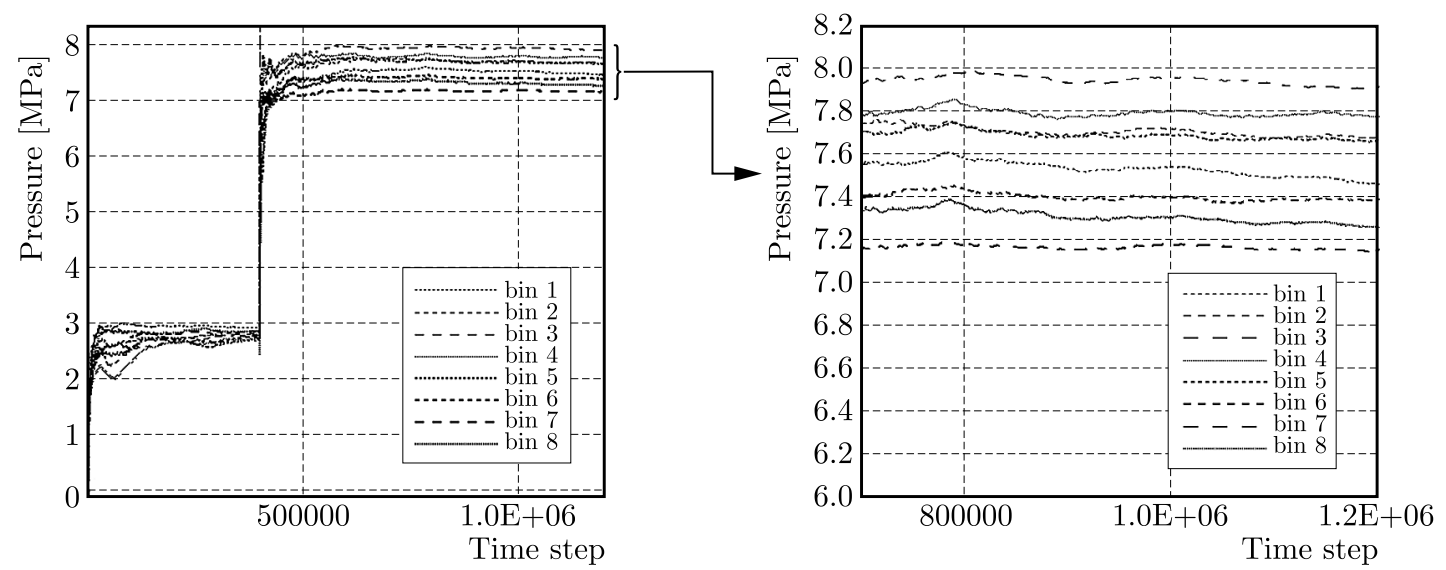

Fig. 5. Convergence of pressure in different bins of the periodic flow with the mean flow velocity of $5 \mathrm{~m} / \mathrm{s}$. A temperature gradient of $30 \mathrm{~K}$ is enforced between bin 3 with $180 \mathrm{~K}$ and bin 7 with $150 \mathrm{~K}$ at the time step 400001

and 7 , respectively. It can also be seen that pressures in other bins are between these two values. In the next Section, results of this case including pressure, density, and velocity gradients in the periodic flow will be discussed in details. At this point, we conclude that pressure can be effectively set to a desired value at anytime or anywhere in the solution domain by setting the temperature of molecules. This finding can be very useful in applying flow boundary conditions in the inlet or the outlet of the solution domain.

\section{Creating a periodic flow with a temperature gradient}

In this Section, we would like to analyze a flow field and gradients of its variables in a periodic flow that is created by a temperature gradient. Consider the flow field from the previous Section that has been created by a temperature gradient enforced between bins 3 and 7 . The mean velocity was set to $5 \mathrm{~m} / \mathrm{s}$ in the third bin, and temperature values of $180 \mathrm{~K}$ and $150 \mathrm{~K}$ were set in bins 3 and 7 , respectively. Having assumed periodic boundary conditions at the inlet and outlet, bins 3 and 7 fall exactly in the middle of the flow field. It means that the distance from bin 3 to bin 7 is equal to the distance from bin 7 to bin 3 in the periodic domain. From the time step 400001, 800000 time steps of $10^{-15} \mathrm{~s}$ are taken to arrive at the equilibrium in the flow field. The implemented temperature gradient of $30 \mathrm{~K}$ has resulted in pressure, mean flow velocity, and molecular density variations shown in Fig. 6. In Fig. 6a, the mean flow velocity decreases from $5 \mathrm{~m} / \mathrm{s}$ in bin 3 to $3.3 \mathrm{~m} / \mathrm{s}$ in bin 7 . Furthermore, pressure has reached its maximum value of $7.9 \mathrm{MPa}$ in bin 3 and its minimum value of $7.15 \mathrm{MPa}$ in bin 7 (Fig. 6b).

(a)

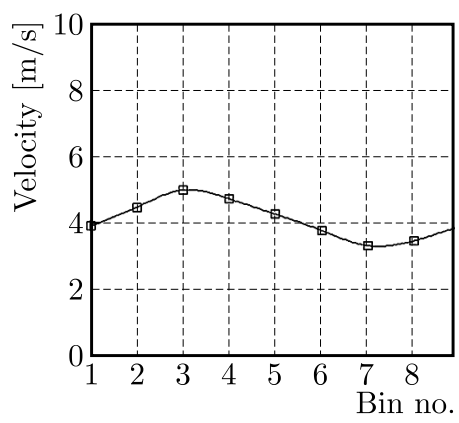

(b)

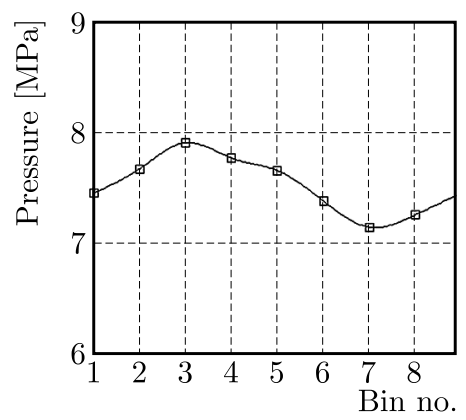

(c)

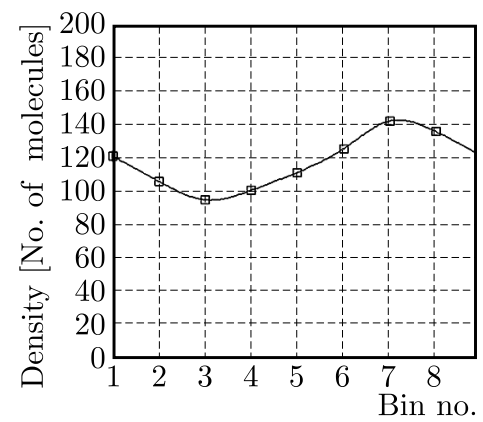

Fig. 6. Variations of flow variables along the periodic flow generated by temperature gradient of $30 \mathrm{~K}$, (a) velocity, (b) pressure and (c) molecular density 
As shown in Fig. 6c, the lowest value of molecular density occurs in bin 3 where temperature value is set to $180 \mathrm{~K}$. This is the highest temperature in the channel. The molecules move with a high virial velocity in this bin and, therefore, they push each other to the outside of the bin. This is why the lowest molecular density occurs here. According to Eq. (3.1), while pressure increases with temperature only through the first term and the effect of molecular density appears in both terms, temperature has the dominant role in determining the pressure value and its variation. Therefore, as seen in Fig. 6b, the highest pressure occurrs at the bin with the highest temperature.

In the next step, we would like to investigate the effect of the temperature gradient on the flow field at different temperature levels. We believe that the temperature level at which the temperature gradient is applied, is important. Variations of velocity, pressure and molecular density for a temperature gradient of $30 \mathrm{~K}$ between bins 3 and 7 , and at different fluid temperature values are shown in Fig. 7. The results are obtained for fixed temperatures of $150 \mathrm{~K}, 180 \mathrm{~K}$, $210 \mathrm{~K}, 240 \mathrm{~K}$, and $270 \mathrm{~K}$ at bin 3 . The mean flow velocity of $5 \mathrm{~m} / \mathrm{s}$ is still forced at bin 3 .
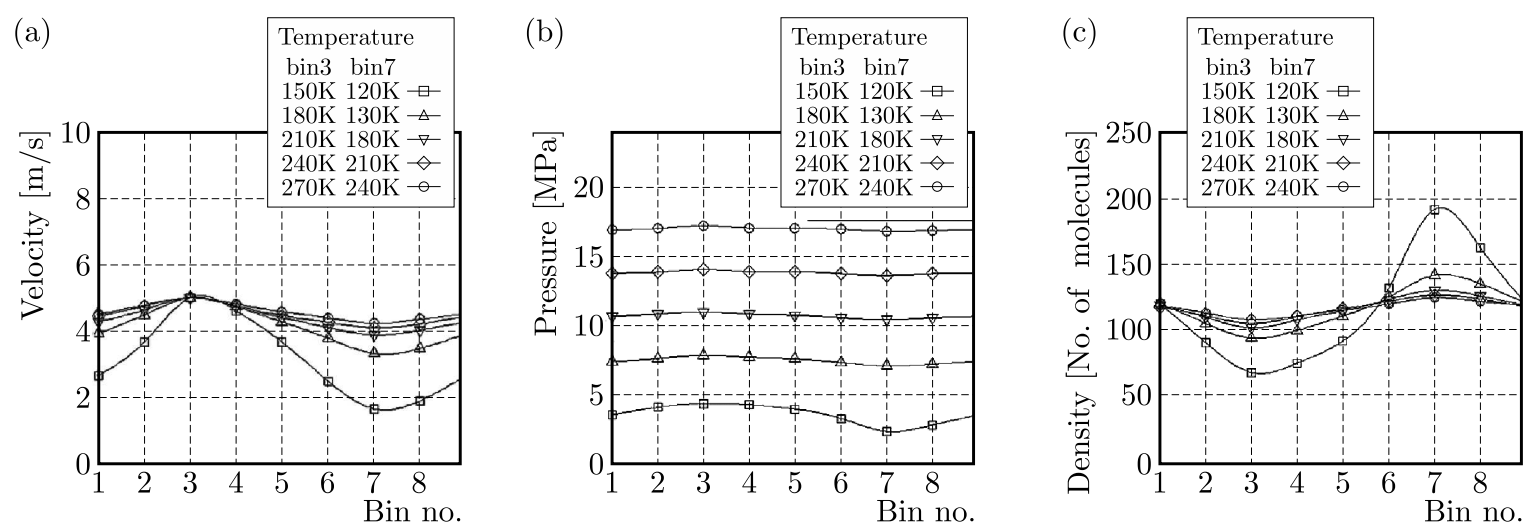

Fig. 7. Variations of flow variables along the channel generated by a temperature gradient of $30 \mathrm{~K}$ at different fluid temperatures, (a) mean flow velocity, (b) pressure and (c) molecular density

In comparison to the level of fluid temperature, the results show that a $30 \mathrm{~K}$ temperature gradient becomes less effective at higher fluid temperature levels. In other words, the $30 \mathrm{~K}$ temperature gradient at the fluid temperature of $150 \mathrm{~K}$ has a higher effect on the flow characters than it does at a fluid temperature of $270 \mathrm{~K}$, for instance. Therefore, it can be seen in Fig. 7 that as the temperature level increases, the mean flow velocity profile and molecular density profile become smoother. In a similar fashion, the pressure profile becomes almost flat at higher temperature values. Furthermore, in Figs. $7 \mathrm{a}$ and $7 \mathrm{c}$, the profiles converge to a single avergage value. This is not the case with the average pressure value which continuously increases with temperature throughout the channel.

From Eq. (3.1), one can conclude that the mean flow velocity will not affect the pressure values since it has no direct contribution in the equation. To study this, the values of temperature in bins 3 and 7 are set to $150 \mathrm{~K}$ and $300 \mathrm{~K}$, respectively enforcing a high temperature gradient of $150 \mathrm{~K}$ in the domain. The molecular flow is solved for this temperature gradient at different mean flow velocities of $5,10,15,20,25,30,35,40$, and 45 meters per second in bin 3 . The profiles of velocity, pressure and molecular density as well as temperature in these experiments are shown in Figs. 8a-8d. In addition, the average temperature value of the molecules in the whole domain has been calculated and plotted in Fig. 8e.

It can be seen in Fig. 8b that pressure decreases uniformly with an increase in the mean flow velocity. This is in contrast with the above-mentioned statement. To further understand the reason behind this behavior, let us analyze the results shown in Fig. 8. From Figs. 8c and 8d, we observe that molecular density is at its maximum in the lower temperature zone of the domain. 


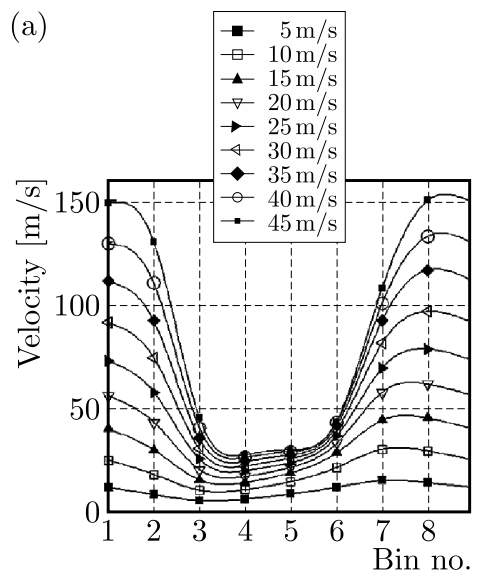

(b)

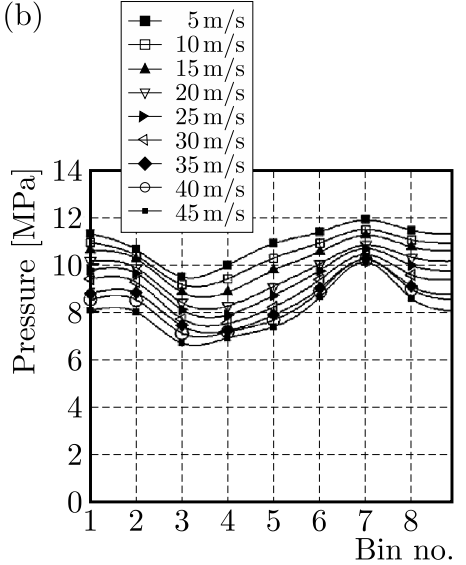

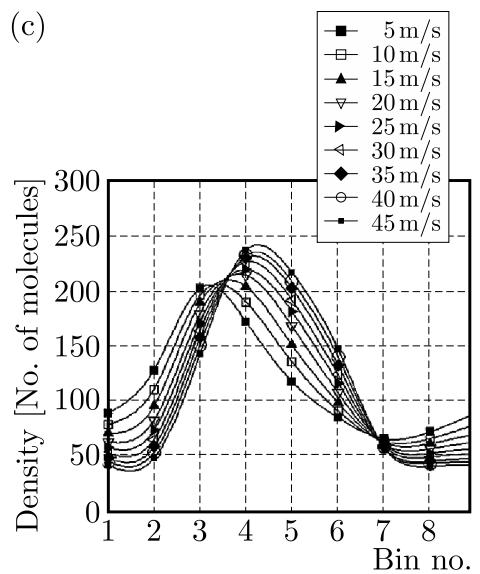

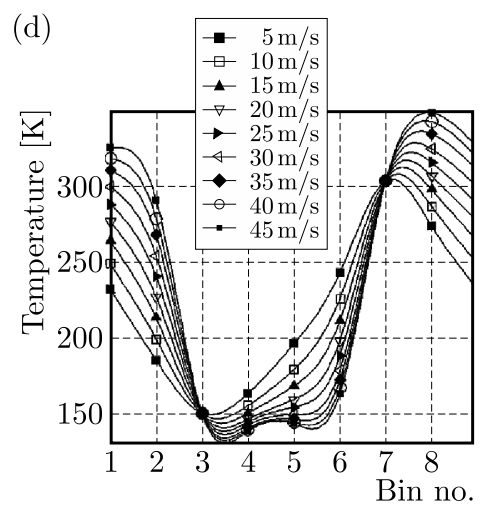

(e)

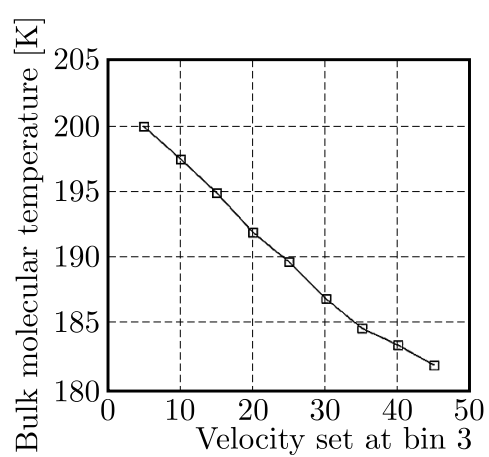

Fig. 8. Variations of flow variables along the channel generated by a temperature gradient of $150 \mathrm{~K}$ at different mean flow velocities. Variations of (a) velocity, (b) pressure and (c) molecular density,

(d) temperature, (e) average temperature of molecules in the whole channel

This trend is more pronounced when the mean flow velocity increases. This means that the number of molecules with lower temperature values increases in the solution domain with the mean flow velocity. As a result, the bulk molecular temperature of the domain decreases with the mean flow velocity (Fig. 8e). Since temperature has the dominant influence on pressure, it can be justified that the reduction of pressure in the domain is a direct result of the reduction in the bulk molecular temperature.

The effect of the temperature gradient on the flow field is also studied here. Different temperature gradient values are examined by setting temperature of bin 7 at $175 \mathrm{~K}, 200 \mathrm{~K}, 225 \mathrm{~K}$, $250 \mathrm{~K}, 275 \mathrm{~K}, 300 \mathrm{~K}, 325 \mathrm{~K}$, and $350 \mathrm{~K}$. A mean flow velocity of $5 \mathrm{~m} / \mathrm{s}$ and temperature of $150 \mathrm{~K}$ are set at bin 3. Variations of velocity, pressure and molecular density for different temperature gradients between bins 3 and 7 are shown in Fig. 9 .

At higher temperature values, the virial velocity of molecules increases and, therefore, molecules repel each other. As a result, the molecular density decreases. In addition, the mean flow velocity of molecules increases since at low molecular density values, fewer obstacles exist on their ways. Based on this argument, the behavior of velocity and density profiles in Figs. 9a and $9 \mathrm{c}$ can be easily justified.

As seen in Fig. 9b, pressure in the whole domain rises with the temperature gradient. An increase in the temperature gradient increases the average temperature of the flow field globally and, therefore, pressure rises in the whole channel. The behavior of pressure profiles can be explained locally as well. In bin 3 for instance, pressure rises due to an increase in molecular density although temperature is constant. In bin 7 , however, pressure rises due to an increase in temperature although molecular density reduces. Once again, note that temperature has the 

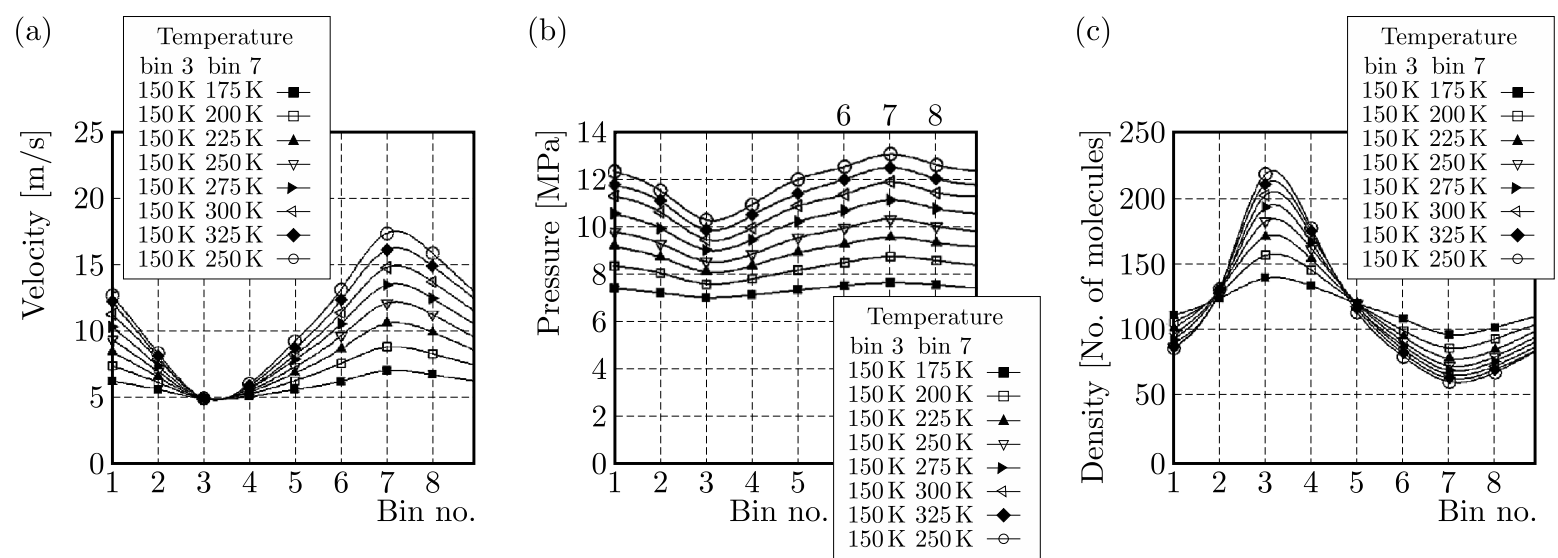

Fig. 9. Variations of flow variables along the channel generated by different temperature gradients at a mean flow velocity of $5 \mathrm{~m} / \mathrm{s}$ in bin 3 . Variations of (a) velocity, (b) pressure and (c) molecular density

primary role in variation of the pressure value and its behavior. Therefore, pressure increases from bin 3 to bin 7 .

The last test case has been repeated with a fixed temperature of $180 \mathrm{~K}$ (instead of $150 \mathrm{~K}$ ) to investigate the flow behavior at a different temperature level. The results are not reported here to limit the length of the present paper; but similar behavior has been observed. At the end, it is worth to mention that while more accurate methods for pressure calculation in inhomogeneous fluids can be found in the literature (Todd et al., 1995), we believe that employment of such methods will not lead to different conclusions than those made herein.

\section{Conclusion}

Details of pressure calculation in a molecular system and its sensitivity to approximations have been studied herein. It has been shown that for molecules near boundaries, inclusion of all molecules within their cut-off region including those outside of the domain boundary is crucial for correct calculation of pressure.

In this paper, a method has been introduced to create a flow by controlling macroscopic properties in two different regions. Temperature values have been imposed at two selected regions along the flow, and the mean flow velocity has been imposed at one of these regions. Having applied these conditions to the periodic flow and conducting parametric study on it, the following results have been obtained.

- Temperature and its gradient have the most dominant role on the variation of the pressure value and its gradient in the periodic flow.

- In the periodic flow, the pressure gradient established as a result of a constant temperature gradient is not always the same at different temperature levels. In fact, both the temperature gradient and the temperature at which this gradient is applied, determine the pressure gradient in the flow.

- Since the mean velocity does not appear in the pressure formula, it is expected that variations of the mean velocity would not change pressure in the periodic flow. However, the results show that for a constant temperature gradient, pressure changes inversely with the mean flow velocity. Based on our analysis, this is because the mean flow velocity directly changes the average temperature of the flow within the channel. 


\section{References}

1. Allen M.P., Tildesley D.J., 1987, Computer Simulation of Liquids Oxford, New York

2. Branam R.D., Micci M.M., 2009, Comparison of wall models for the molecular dynamics simulation of microflows, Nanoscale and Microscale Thermophysical Engineering, 13, 1-12

3. Darbandi M., Abbassi H.R., Khaledi Alidusti R., Sabouri M., 2011, Molecular dynamics simulation of nano channel as nanopumps, ICNMM, Edmonton, Alberta, Canada

4. Fan X.J., Phan-Thien N., Teng Yong N., Diao X., 2002, Molecular dynamics simulation of a liquid in a complex nano channel flow, Physics of Fluids, 14, 3, 1146-1153

5. HAN M., 2008, Thermally-driven nanoscale pump by molecular dynamics simulation, Journal Title: Journal of Mechanical Science and Technology, 22, 157-165

6. Hanasaki I., NakAtani A., 2006, Fluidized piston model for molecular dynamics simulations of hydrodynamics flow, Modelling and Simulation in Materials Science and Engineering, 14, S9-S20

7. Huang C., Choi P.Y.K., Nandakumar K., Kostiuk L.W., 2006, Molecular dynamics simulation of a pressure-driven liquid transport process in a cylindrical nanopore using two self-adjusting plates, Journal of Chemical Physics, 124, 234701

8. Huang C., Nandakumar K., Kwok D.Y., 2004, Non-equilibrium injection flow in a nanometer capillary channel, ICMENS'04, 374-378

9. Kamali R., Kharazmi A., 2011, Molecular dynamics simulation of surface roughness effects on nanoscale flows, International Journal of Thermal Sciences, 50, 3, 226-232

10. Karniadakis G.E., Beskok A., Aluru N., 2002, Micro Flows and Nanoflows, Springer, New York, 641-648

11. Karimian S.M.H., Izadi S., 2013, Bin size determination for the measurement of mean flow velocity in molecular dynamics simulation, International Journal For Numerical Methods In Fluids, 71, 7, 930-938

12. Karimian S.M.H., Izadi S., Barati FArimani A., 2011, A study on the measurement of mean velocity and its convergence in molecular dynamics simulations, International Journal for Numerical Methods in Fluids, 67, 12, 2130-2140

13. Karimian S.M.H., NAmvar S., 2012, Implementation of SMC averaging method in a channeled molecular flow of liquids and gases, Journal of Physics: Conference Series, 362, 1, 2029

14. Kim B.H., Beskok A., CAgin T., 2010, Viscous heating in nanoscale shear driven liquid flows, Microfluid Nanofluid, 9, 31-40

15. Koplik J., Banavar J., Willemsen J., 1988, Molecular dynamics of poiseuille flow and moving contact lines, Physical Review Letters, 60, 1282-1285

16. Koplik J., Banavar J. R., Willemsen J.F., 1989, Molecular dynamics of fluid flow at solid surfaces, Physics of Fluids A, 1, 781-794

17. Leach A.R., 1999, Molecular Modeling: Principles and Applications, Longman

18. LiU C., Li Z., 2010, Molecular dynamics simulation of composite nanochannels as nanopumps driven by symmetric temperature gradients, Physical Review Letters, 105, 174501

19. Mi X.B., Chwang A. T., 2003, Molecular dynamics simulations of nanochannel flows at low Reynolds numbers, Molecules, 8, 193-206

20. Nagayama G., Cheng P., 2004, Effects of interface wettability on microscale flow by molecular dynamics simulation, International Journal of Heat and Mass Transfer, 47, 501-513

21. Namvar S., Karimian S.M.H., 2012, Detailed investigation on the effect of wall spring stiffness on velocity profile in molecular dynamics simulation, Journal of Physics: Conference Series, 362, 1,2039 
22. Plimpton S.J., 1995, Fast parallel algorithms for short-range molecular dynamics, Journal of Computational Physics, 117, 1

23. PriezJeV N.V., 2007, Effect of surface roughness on rate-dependent slip in simple fluids, Journal of Chemical Physics, 127, 144708

24. Rapaport D.C., 2004, The Art of Molecular Dynamics Simulation, Cambridge University Press

25. Sadus R.J., 2002, Molecular Simulation of Fluids: Theory, Algorithms and Object-Orientation, Elsevier

26. Sofos F., Karakasidas T.E., Liakopoulos A., 2009, Non-equilibrium molecular dynamics investigation of parameters affecting planar nanochannel flows, Contemporary Engineering Sciences, 2, 6, 283-298

27. Stillinger F.H., Rahman A., 1974, Improved simulation of liquid water by molecular dynamics, Journal of Chemical Physics, 60, 1545-1557

28. Sun M., Ebner C., 1992, Molecular-Dynamics simulation of compressible fluid flow in two-dimensional channels, Physical Review A, 46, 4813

29. Todd B.D., Evans D.J., Davis P.J., 1995, Pressure tensor for inhomogeneous fluids, Physical Review E, 52, 1627

30. Travis K.P., Evans D.J., 1997, Molecular spin in a fluid undergoing Poiseuille flow, Physical Review E, 55, 1566-1572

31. Travis K.P., Gubbins K.E., 2000, Poiseuille flow of Lennard-Jones fluids in narrow slit pores, Journal of Chemical Physics, 112, 1984-1994

32. Tysanner M.W., Garcia A.L., 2004, Measurement bias of fluid velocity in molecular simulations, Journal of Computational Physics, 196, 173-183

33. Tysanner M.W., Garcia A.L., 2005, Non-equilibrium behavior of equilibrium reservoirs in molecular simulations, International Journal of Numerical Methods in Fluids, 2050, 1-12

34. Verlet L., 1967, Computer "experiments" on classical fluids. I. Thermodynamical properties of Lennard-Jones molecules, Physical Review, 159, 98

35. Zhang Z.Q., Zhang H.W., Ye H.F., 2009, Pressure-driven flow in parallel-plate nanochannels, Applied Physics Letters, 95, 154101

36. Ziarani A.S., Mohamad A.A., 2005, A Molecular dynamics study of perturbed Poiseuille flow in a nanochannel, Microfluid Nanofluid, 2, 12-20 